\title{
Maturation of primary and permanent teeth in preterm infants
}

M C Backström, L Aine, R Mäki, A-L Kuusela, H Sievänen, A-M Koivisto, R-S Ikonen, M Mäki

\begin{abstract}
Aims-To elucidate the development of primary and permanent teeth and to interpret the effect of different calcium, phosphorus, and vitamin D supplementation in the neonatal period on dental maturation in preterm children.

Methods-Preterm infants were randomised to four groups to receive a vitamin $D$ dose of 500 or 1000 IU/day and calcium and phosphorus supplemented or unsupplemented breast milk. The maturity of the primary and permanent teeth was recorded in 30 preterm children. Sixty children aged 2 years and 60 children aged 9-11 years served as controls. Bone mineral content/density was assessed in the preterm infants.
\end{abstract}

Results-The median (range) corrected teething age was $7(2-16)$ months in preterm infants and $6(2-12)$ months in controls $(p=0.43)$. The median (range) number of erupted teeth at 2 years of age was 16 (11-19) in preterm infants and 16 $(12-20)$ in controls $(p=0.16)$. Maturation of the permanent teeth in the preterm infants was not delayed compared with the controls (mean Demirjian SDS $0.16 v 0.49$, $p=0.14)$. Early dietary intake of either mineral or vitamin $D$ did not affect maturation of the primary dentition in preterm children. Children receiving the higher vitamin $\mathrm{D}$ dose in the neonatal period had more mature permanent dentition than those receiving the lower dose, but mineral intake did not affect maturation of the permanent teeth. Dental maturation did not correlate with bone mineral status.

Conclusions-This is the first longitudinal study to follow primary and permanent tooth maturation in the same preterm children. Premature birth has no appreciable late sequelae in tooth maturation.

(Arch Dis Child Fetal Neonatal Ed 2000;83:F104-F108)

Keywords: dental maturation; preterm; calcium; vita$\min \mathrm{D}$; teeth

Tooth maturation can be delayed in systemic disorders such as hypothyroidism. ${ }^{1}$ There are limited data for preterm children on the development of primary ${ }^{2-4}$ and permanent teeth. ${ }^{5}$ The effects of early dietary mineral and vitamin $\mathrm{D}$ intake on bone mineral status in preterm infants have been extensively explored. ${ }^{6-11}$ It is well known that breast milk contains too little calcium and phosphorus to enable intrauterine mineral accretion in preterm infants. Thus the main cause of metabolic bone disease of prematurity is an inadequate supply of calcium and phosphorus, not vitamin $\mathrm{D}$ deficiency, as long as a dose of $160-1000 \mathrm{IU} /$ day is used as a supplement. ${ }^{6-11}$ However, the effect of calcium, phosphorus, and vitamin D supplementation on dental maturation in preterm infants has not been reported. The aim of this study was to define the development of primary and permanent teeth in the same preterm infants and to investigate the effect of different calcium, phosphorus, and vitamin $\mathrm{D}$ supplementations in the neonatal period on dental maturation in children born preterm. A further objective was to investigate whether tooth development is associated with bone mineral status in preterm children.

\section{Methods}

Between August 1985 and May 1987, a cohort of preterm infants with a birth weight less than $2000 \mathrm{~g}$ were investigated to evaluate the effect of early dietary intake of mineral and vitamin $\mathrm{D}$ on bone mineral accretion and dental development. The study was conducted at the neonatal intensive care unit in Tampere University Hospital. The inclusion criterion was a gestational age of less than 37 weeks at birth. The exclusion criterion was any major congenital abnormality. The withdrawal criterion was failure of vitamin D administration according to the protocol (see below); in fact, there were no withdrawals. During the study period, the clinical practice in our hospital was to give all preterm infants unsupplemented breast milk and vitamin D $1000 \mathrm{IU} /$ day. According to the study protocol, all preterm infants enrolled in the study were stratified according to birth weight and then randomly assigned to one of four groups to receive vitamin D supplementation either 500 or $1000 \mathrm{IU} /$ day from the time of tolerance of full enteral nutrition until 6 months of chronological age, and to receive either unsupplemented breast milk $(\mathrm{CaP}-)$ or breast milk supplemented with calcium 108 $\mathrm{mg} / \mathrm{kg} /$ day and phosphorus $53 \mathrm{mg} / \mathrm{kg} /$ day $(\mathrm{CaP}+)$ from the time of tolerance of full enteral nutrition until they reached a body weight of $2000 \mathrm{~g}$. Primary dentition was examined at age 1 and 2 during routine visits in 30 children (16 girls and 14 boys). Permanent dentition was examined in the same preterm children at age 9-11. The controls were 60 healthy 2 year old children and 60 healthy children aged 9-11, all of whom had been born at full term; both groups of controls were matched for age and sex with the study children. Bone mineral content (BMC) was 
assessed by single photon absorptiometry at 3 months of age in eight of the preterm infants. At 9-11 years, bone mineral density (BMD) was measured by dual energy $x$ ray absorptiometry in 29 of the preterm children. Plasma 25-hydroxyvitamin D (25(OH)D) and 1,25dihydroxyvitamin $\mathrm{D}\left(1,25(\mathrm{OH})_{2} \mathrm{D}\right)$ concentrations were measured at 3 and 6 months of chronological age in the preterm infants. The median birth weight of the preterm infants was 1505 (range 690-1930) $\mathrm{g}$ and the median gestational age 31.5 (range 23.7-35.0) weeks. Two of these children suffered from mild spastic diplegia, and three from bronchial asthma. The remaining 25 did not have any chronic illnesses. The group allocation was concealed from the dentist and those measuring plasma vitamin D metabolite concentrations and bone density.

At a chronological age of 3 months, body weight and length were obtained from clinical charts. At 9-11 years of age, body weight and height were measured and pubertal status determined. Body weight was determined with a Secca Delta electronic scales model 707 and height with a Harpender stadiometer.

DENTAL EXAMINATIONS

All dental examinations were performed by one investigator (LA) at the Oral Department in the Department of Otorhinolaryngology and Maxillofacial Surgery in the University Hospital of Tampere. The examinations were carried out using an artificial light in a dental chair.

\section{Primary dentition}

Maturity in the primary dentition was recorded in 30 preterm children at the ages of 1 and 2 during routine visits and in 60 control children at the age of 2 . Maturity was assessed by counting the erupted teeth. A tooth was considered to be clinically erupted when the crown or part of it had penetrated the mucous membrane. Both chronological and corrected teething age (corrected teething age $=$ chronological teething age in weeks - $(40-$ gestational age at birth in weeks)) were recorded. The age when the first primary tooth erupted was recorded from well baby clinic records in both preterm children and controls.

\section{Permanent dentition}

Maturity of the permanent dentition was recorded in the same 30 preterm children at the age of 9-11 years and in 60 control children. Dental maturity was assessed from panoramic radiographs using the method of Demirjian $e t a l^{12}$ and updated by Demirjian and Goldstein. ${ }^{13}$ In this method dental maturity is based on seven left mandibular teeth, the developmental stage of which is evaluated according to criteria given by Demirjian. The summed scores of all seven teeth give the dental maturity score, which can be converted into dental age by using the curves given by Demirjian and Goldstein. ${ }^{13}$

BONE DENSITOMETRY

At the age of 3 months, linear BMC $(\mathrm{g} / \mathrm{cm})$ was determined by single photon absorptiometry based on $28 \mathrm{keV} \gamma$ radiation from an ${ }^{125}$ I source in eight of the infants. ${ }^{14}{ }^{15} \mathrm{~A}$ piece of apparatus constructed in house consisting of source, collimated radiation detector, and plotter was used for measurements. Two repeated single photon absorptiometry scans were taken at the distal site of the left forearm, specifically at one third of the ulnar length measured proximally from the distal ulnar styloid. The left forearm was placed between two rubber gloves filled with water. The mean of two BMC measurements was used.

At the age of 9-11, BMD was measured by dual energy $x$ ray absorptiometry (Norland XR-26; Norland Corp, Fort Atkinson, Wisconsin, USA) in the lumbar spine (L2-L4) of 29 participants. Measurement and analysis procedures are described in detail elsewhere. ${ }^{15}$ All scans and analyses were performed by the same experienced laboratory technician in a blinded fashion. The scanner was calibrated daily and its performance monitored by our quality assurance protocol. ${ }^{16}$ In addition to standard area BMD $\left(\mathrm{g} / \mathrm{cm}^{2}\right)$ measurements, a volumetric bone mineral apparent density (BMAD, $\mathrm{g} / \mathrm{cm}^{3}$ ) in the lumbar spine was calculated by assuming the shape of vertebral bodies to be cylindrical. ${ }^{17}$ The in vivo precision (coefficient of variation) of the above measurements in our laboratory is about $1 \% .^{17}$

DETERMINATION OF PLASMA VITAMIN D METABOLITES

$25(\mathrm{OH}) \mathrm{D}$ and $1,25(\mathrm{OH})_{2} \mathrm{D}$ were measured at chronological age 3 and 6 months using $1 \mathrm{ml}$ plasma samples to which $\left[{ }^{3} \mathrm{H}\right] 25(\mathrm{OH}) \mathrm{D}_{3}$ and $\left[{ }^{3} \mathrm{H}\right] 1,25(\mathrm{OH})_{2} \mathrm{D}_{3}$ were added to monitor recovery throughout the assay. The samples were deproteinised and purified using the acetonitrile- $\mathrm{C}_{18}$ Sep-Pak method. ${ }^{18}$ Thereafter the metabolites were further purified and separated by high performance liquid chromatography. A LiChrosorb Si $605(\mathrm{~m})$ column (E Merck, Darmstadt, Germany) eluted with hexane/dichloromethane/methanol/propan-2-ol (76:16:5:3, by vol) was used. $25(\mathrm{OH}) \mathrm{D}$ was measured by a method based on binding to the competing protein, ${ }^{19}$ using serum from a pregnant woman diluted 1:20000 in barbital acetate buffer, $\mathrm{pH}$ 8.6, and $\left[{ }^{3} \mathrm{H}\right] 25(\mathrm{OH}) \mathrm{D}$. Non-radioactive $25(\mathrm{OH}) \mathrm{D}$ served as the standard. $1,25(\mathrm{OH})_{2} \mathrm{D}$ was measured by a radioreceptor method. ${ }^{20}$ Interassay and intra-assay coefficients of variation for each of the metabolites ranged from 11.7 to $14.5 \%$. The paediatric reference range for $25(\mathrm{OH}) \mathrm{D}$ in our laboratory is $30-130 \mathrm{nmol} / 1$, and in the winter the lower limit is $17.5 \mathrm{nmol} / \mathrm{l}$. The laboratory reference range for $1,25(\mathrm{OH})_{2} \mathrm{D}$ is $48-175$ $\mathrm{pmol} / 1$.

\section{STATISTICAL ANALYSIS}

The data were analysed using the statistical software SPSS Windows version 6.1 (SPSS Inc, Chicago, Illinois, USA). For variables with skewed distributions, median and range were given as descriptive statistics, and the differences between the groups were tested by the Mann-Whitney U test or Kruskall-Wallis nonparametric analysis of variance when appropriate. Means (SD) were given as descriptives for 
Table 1 Development of deciduous teeth in children born preterm and at term

\begin{tabular}{|c|c|c|c|c|c|c|c|c|c|c|}
\hline & \multicolumn{4}{|c|}{ Girls } & \multirow[b]{3}{*}{$p$ Value $^{\star}$} & \multicolumn{4}{|c|}{ Boys } & \multirow[b]{3}{*}{$p$ Value ${ }^{\star}$} \\
\hline & \multicolumn{2}{|c|}{ Preterm } & \multicolumn{2}{|c|}{ Term } & & \multicolumn{2}{|c|}{ Preterm } & \multicolumn{2}{|c|}{ Term } & \\
\hline & $n$ & Median (range) & $n$ & Median (range) & & $n$ & Median (range) & $n$ & Median (range) & \\
\hline $\begin{array}{l}\text { Chronological age at eruption } \\
\text { of first tooth (months) }\end{array}$ & $15 \dagger$ & $9(5-17)$ & 32 & $6(3-12)$ & $<0.01$ & $15+$ & $7(6-15)$ & 28 & $6(2-10)$ & $<0.01$ \\
\hline $\begin{array}{l}\text { Corrected age at eruption } \\
\text { of first tooth (months) }\end{array}$ & $15 \dagger$ & $8(3-16)$ & 32 & $6(3-12)$ & 0.02 & $13+$ & $5(2-12)$ & 28 & $6(2-10)$ & 0.18 \\
\hline Number of teeth at age 2 years & 16 & $16(11-18)$ & 32 & $16(12-20)$ & 0.22 & 14 & $16(15-19)$ & 28 & $16(12-20)$ & 0.52 \\
\hline Demirjian SDS & 22 & $0.30(-2.41-1.48)$ & 109 & $0.42(-0.46-1.74)$ & & 18 & $0.27(-1.24-1.88)$ & 95 & $0.33(-0.66-2.62)$ & 0.46 \\
\hline
\end{tabular}

*Mann-Whitney U test.

$t_{n}=15+13=28$; information was missing on eruption of first tooth in two children.

Table 2 Development of deciduous teeth in children born preterm who received mineral supplemented (CaP+) or unsupplemented (CaP-) breastmilk and vitamin D 500 IU/day or 1000 IU/day in the neonatal period

\begin{tabular}{|c|c|c|c|c|c|c|c|c|c|}
\hline & \multicolumn{4}{|c|}{$\mathrm{CaP}+$} & \multicolumn{4}{|c|}{$\mathrm{CaP}-$} & \multirow[b]{3}{*}{$p$ Value ${ }^{\star}$} \\
\hline & \multicolumn{2}{|c|}{500 IU/day } & \multicolumn{2}{|c|}{1000 IU/day } & \multicolumn{2}{|c|}{500 IU/day } & \multicolumn{2}{|c|}{1000 IU/day } & \\
\hline & $n$ & $\begin{array}{l}\text { Median } \\
\text { (range) }\end{array}$ & $n$ & $\begin{array}{l}\text { Median } \\
\text { (range) }\end{array}$ & $n$ & $\begin{array}{l}\text { Median } \\
\text { (range) }\end{array}$ & $n$ & $\begin{array}{l}\text { Median } \\
\text { (range) }\end{array}$ & \\
\hline Chronological age at eruption of first tooth (months) & 4 & $10(6-15)$ & 4 & $9(7-10)$ & 11 & $8(6-17)$ & 9 & $9(5-11)$ & 0.93 \\
\hline Corrected age at eruption of first tooth (months) $\dagger$ & 4 & $7(3-12)$ & 4 & $6(6-8)$ & 11 & $6(2-16)$ & 9 & $7(3-8)$ & 0.91 \\
\hline Number of teeth at 1 year of age $\neq$ & 4 & $4(0-8)$ & 4 & $4(1-8)$ & 11 & $5(0-8)$ & 10 & $6(1-8)$ & 0.75 \\
\hline Number of teeth at 2 year of age & 4 & $16(16-17)$ & 5 & $16(14-18)$ & 11 & $16(11-18)$ & 10 & $16(15-19)$ & 0.99 \\
\hline
\end{tabular}

$\star$ Kruskall-Wallis non-parametric analysis of variance.

$t_{\mathrm{n}}=4+4+11+9=28$; information missing on eruption of first tooth in two children.

$\ddagger \mathrm{n}=4+4+11+10=29$; data missimg on number of teeth at 1 year of age in one child.

the variables with normal distribution, and they were tested by Student's $t$ test or two way analysis of variance when appropriate. Spearman's correlation coefficient $(r)$ was used to evaluate the strength of association between selected quantitative variables. The $\alpha$ level was set at 0.05 .

ETHICAL CONSIDERATIONS

The study was approved by the ethical committee of Tampere University Hospital, and written informed consent was obtained from the parents.

\section{Results}

PRIMARY DENTITION

The median (range) chronological age when the first tooth erupted - that is, teething agewas 9 (5-17) months in the preterm infants. The corrected teething age was $7(2-16)$ months, which corresponds well to that of the control children: $6(2-12)$ months $(p=0.43)$. At a chronological age of 1 year, $5(0-8)$ teeth (median (range)) had erupted in the preterm infants. At a chronological age of 2 years, the corresponding number was $16(11-19)$ teeth in preterm children and $16(12-20)$ in the controls $(p=0.16)$. Maturation of primary teeth was thus not significantly delayed in the whole study group of preterm children. However, when the preterm children were divided according to sex, the corrected age at the time of eruption of the first tooth in preterm girls was significantly later than in control girls, whereas the teething age was not delayed in preterm boys (table 1). Furthermore, eruption of the first tooth occurred significantly later in preterm girls than in preterm boys $(p=0.01)$, whereas no difference between boys and girls was seen in term children $(p=0.64)$. At 2 years of age, the median number of erupted teeth was 16 in both preterm and control boys and girls (table 1). There was no association between gestational age at birth $(r=0.14$, $\mathrm{p}=0.31)$ or birth weight $(r=-0.23$, $\mathrm{p}=0.08)$ and the corrected age at eruption of the first tooth. However, the heavier the infant at 3 months chronological age, the sooner the first tooth erupted $(r=-0.68, \mathrm{p}<0.01)$. The age when full enteral nutrition was tolerated $(r=-0.02, \mathrm{p}=0.88)$ and the length of tracheal intubation $(r=0.04, \mathrm{p}=0.77)$ were not associated with the corrected teething age. Late teething correlated with fewer erupted teeth at 1 and 2 years of chronological age $(r=-0.57, \mathrm{p}<0.01$ and $r=-0.34, \mathrm{p}=0.03)$ in the preterm children.

The median serum $25(\mathrm{OH}) \mathrm{D}$ concentration at 3 and 6 months chronological age did not differ between infants receiving vitamin D 500 $\mathrm{IU} /$ day or $1000 \mathrm{IU} /$ day in the neonatal period (3 months: $143.5 v 174.5 \mathrm{nmol} / \mathrm{l}, \mathrm{p}=0.31 ; 6$ months: 147.4 v $172.0 \mathrm{nmol} / \mathrm{l}, \mathrm{p}=0.18$ ), neither was the median serum $1,25(\mathrm{OH})_{2} \mathrm{D}$ concentration (3 months: $243.0 v 160.0$ $\mathrm{nmol} / \mathrm{l}, \mathrm{p}=0.09 ; 6$ months: $282.0 \vee 195.0$ $\mathrm{nmol} / \mathrm{l}, \mathrm{p}=0.09)$.

Early dietary vitamin D supplementation did not affect maturation of the primary teeth (table 2). Furthermore, early dietary intake of vitamin $\mathrm{D}$ or mineral did not affect the chronological or corrected age at eruption of the first tooth or the number of erupted teeth at 1 and 2 years of chronological age (table 2). There was no association between BMC at 3 months of chronological age and maturation of primary dentition $(r=-0.30$ to 0.31 , $\mathrm{p}=0.32-0.88)$. There was no difference in birth weight, gestational age, duration of tracheal intubation, age at tolerance of full enteral nutrition, or age when body weight reached $2000 \mathrm{~g}$ between the four feeding groups (data not shown). 
Table 3 Group characteristics and development of permanent teeth at 9-11 years of age in children born preterm who received mineral supplemented (CaP+) or unsupplemented (CaP-) breastmilk and vitamin D 500 IU/day or 1000 IU/day in the neonatal period

\begin{tabular}{|c|c|c|c|c|c|}
\hline & \multicolumn{2}{|l|}{$\mathrm{CaP+}$} & \multicolumn{2}{|l|}{$\mathrm{CaP}-$} & \multirow[b]{2}{*}{$p$ Value ${ }^{\star}$} \\
\hline & 500 IU/day $(n=4)$ & $1000 \mathrm{IU} /$ day $(n=5)$ & $500 \mathrm{IU} /$ day $(n=11)$ & 1000 IU/day $(n=10)$ & \\
\hline Age (years) & $10.7 \quad(9.5-10.8)$ & $10.5(9.7-10.7)$ & $10.7 \quad(8.9-11.3)$ & $10.9 \quad(9.7-11.7)$ & 0.24 \\
\hline Weight (kg) & $28.6 \quad(22.8-35.1)$ & $31.5(21.0-35.0)$ & $32.2(20.7-70.7)$ & $39.0 \quad(26.5-56.8)$ & 0.22 \\
\hline Height $(\mathrm{cm})$ & $140.9(129.0-144.7)$ & $134.3(129.5-150.3)$ & $144.0 \quad(126.3-162.2)$ & $145.2(132.5-151.7)$ & 0.50 \\
\hline Demirjian SDS & $-0.12(-1.31-1.29)$ & $1.0(-1.67-1.48)$ & $-0.20(-2.42-1.18)$ & $0.64(-0.88-1.88)$ & 0.14 \\
\hline
\end{tabular}

Values are median (range).

$\star$ Kruskall-Wallis non-parametric analysis of variance.

PERMANENT DENTITION

Maturation of the permanent teeth in the whole group of children born preterm did not differ from that in the controls born at term (mean Demirjian SDS $0.16 v 0.49, \mathrm{p}=0.14$ ). When the children were divided according to sex, neither the preterm girls (mean Demirjian SDS $0.08 v 0.58, \mathrm{p}=0.12$ ) nor the preterm boys (mean Demirjian SDS $0.26 v 0.38$, $\mathrm{p}=0.74$ ) differed from the term ones. The Demirjian SDS was more advanced, the taller the preterm children were at the time of evaluation of dental status $(r=0.42, \mathrm{p}=0.02)$. Maturation of the permanent teeth did not depend on body weight $(r=0.26, \mathrm{p}=0.15)$ or pubertal stage $(r=0.26, \mathrm{p}=0.16)$. If teething was late and maturation of the primary teeth at 1 year of chronological age was slow, maturation of the permanent dentition at 9-11 years of age was also slow. However, no association was found between the number of teeth at 2 years of chronological age and the Demirjian SDS at 9-11 years of age $(r=0.13, \mathrm{p}=0.36)$. Neither low birth weight $(r=0.17, \mathrm{p}=0.36)$ nor gestational age at birth $(r=0.08, \mathrm{p}=0.68)$ was associated with maturation of the permanent dentition.

Surprisingly, the most mature permanent teeth were found in the preterm children who had received vitamin D 1000 IU/day, whereas mineral supplementation did not affect maturation (table 3 ). This impact was still seen after the effect of body weight had been eliminated by analysis of covariance. Maturation of the permanent teeth in the preterm children was not associated with lumbar bone mineral status at $9-11$ years of age $(r=0.10, p=0.72$ for $\mathrm{BMD}$ and $r=-0.05, \mathrm{p}=0.86$ for $\mathrm{BMAD})$. The prematurely born children receiving vitamin D $500 \mathrm{IU} /$ day and those who had received $1000 \mathrm{IU} /$ day had comparable BMD at 9-11 years of age (mean $0.615 \mathrm{~g} / \mathrm{cm}^{2} v 0.632 \mathrm{~g} / \mathrm{cm}^{2}$ $(\mathrm{p}=0.61)$ for lumbar BMD and mean 0.104 $\mathrm{g} / \mathrm{cm}^{3} v 0.108 \mathrm{~g} / \mathrm{cm}^{3}(\mathrm{p}=0.47)$ for lumbar BMAD).

\section{Discussion}

No prospective studies such as the present one, monitoring dental maturation of both primary and permanent dentition within the same cohort of prematurely born children, have to our knowledge been previously reported. The preterm infants as a whole study group did not have a delayed corrected teething age. This is in accordance with the findings of Seow and coworkers $^{2}$ and Golden and coworkers, ${ }^{3}$ who showed that the chronological teething age in children born preterm was delayed, but not the corrected teething age. It is noteworthy that the preterm girls in our study had their first tooth a median of two months later than girls born at term, whereas the corrected teething age of boys was not delayed. This is in agreement with a study in which male advancement was observed in the development of primary teeth during the first trimester, ${ }^{4}$ whereas, after the first postnatal year, tooth formation is more advanced in girls. ${ }^{18-21}$

The results of the present study imply that different intakes of vitamin $\mathrm{D}$ and mineral in the neonatal period does not affect maturation of the deciduous teeth in children born preterm. It is well known that calcium and phosphorus supplementation in preterm infants in the neonatal period increases bone mineral accretion during the first few years. ${ }^{6-8}$ In the present study, BMC at 3 months of chronological age was not associated with maturation of the primary dentition. This is also in agreement with previous findings that eruption of the primary teeth was associated with somatic growth ${ }^{21}$ but not with skeletal maturity. ${ }^{22}$ There is moreover evidence that the chronology of the eruption of primary teeth is quite extensively genetically determined. ${ }^{23}{ }^{24}$

Seow and coworkers ${ }^{5}$ have reported that maturation of the permanent dentition in children born preterm is delayed at 6 years of age but that at 9 years of age it has caught up. This corresponds well to the results of our study, in which permanent teeth at 9-11 years of age were as mature in children born preterm as in controls. The mean Demirijian SDS of the control children in this study was 0.49 , similar to that of children in other Finnish studies. ${ }^{25} 26$ In the present study, it is shown for the first time that a small number of primary teeth at 1 year of chronological age is associated with less mature permanent teeth at 9-11 years of age in children born preterm. Similarly, in children born at term, a weak significant correlation between maturation of the primary and permanent dentition has been observed. ${ }^{2127}$

This study indicates that maturation of permanent teeth is not affected by early dietary mineral intake. Interestingly, the preterm children receiving vitamin D $1000 \mathrm{IU} /$ day had more mature permanent teeth at 9-11 years of age than those who had received vitamin D $500 \mathrm{IU} /$ day. We suggest that the smaller vitamin $\mathrm{D}$ dose per se is not likely to have impeded the maturation of the permanent teeth, as there was no intergroup difference in the $25(\mathrm{OH}) \mathrm{D}$ concentrations at 3 or 6 months. 
Even considerably smaller vitamin $\mathrm{D}$ doses than 500 IU/day have been reported to maintain the normal vitamin D status in the neonatal period. ${ }^{1128}$ Moreover, giving a large vitamin $\mathrm{D}$ dose to preterm infants may have detrimental effects on bone mineralisation early in life. ${ }^{29}$

In conclusion, bearing in mind the small number of subjects, early dietary mineral intake did not affect maturation of the primary or permanent dentition in children born preterm. Early dietary vitamin D intake had no impact on maturation of the primary dentition. Surprisingly, the children who had received the higher vitamin D dose of $1000 \mathrm{IU} /$ day in the neonatal period had more mature permanent dentition than those who had received the smaller dose of $500 \mathrm{IU} /$ day. This, however, needs further verification. Taking the group as a whole, maturation of both primary and permanent dentition in children born preterm does not differ appreciably from that in children born at term, indicating that premature birth has no appreciable late sequelae with respect to tooth maturation.

1 Mgángá PM, Chiandia ML. Dental and skeletal changes in uvenile hypothyroidism following treatment: case report. Odonto-Stomatologie Tropicale 1990;13:25-7.

2 Seow WK, Humphrys C, Mahonda R, Tudehope DI. Dental eruption in low birth weight prematurely-born low-weight children: a controlled study. Pediatr Dent 1988; 10:39-42.

3 Golden NL, Takieddine F Hirsch VJ. Teething age in prematurely born infants. American fournal of Diseases in Children 1981;135:903-4.

4 Garn SM, Burdi AR. Prenatal ordering and postnatal sequence in dental development. F Dent Res
1971;50:1407-14.

5 Seow K. A study of the development of the permanent dentition in very low birth weight children. Pediatr Dent 1996;18:379-84.

6 Horsman A, Ryan SW, Truscott JG. Bone mineral content and body size 65 to 100 weeks' postconception in preterm and fullterm infants. Arch Dis Child 1985;64:1579-86.

7 Bishop NJ, King FJ, Lucas A. Increased bone mineral content of preterm infants fed with a nutrient enriched formula after discharge from hospital. Arch Dis Child 1993;68:573-8.

8 Schanler RJ, Abrams SA. Postnatal attainment of intrauterine macromineral accretion rates in low birth weigh infants fed fortified human milk. F Pediatr 1995;26:441-7.

9 Pittard WB III, Geddes KM, Husley TC, Hollis WB. How much vitamin $\mathrm{D}$ for neonates? Am $\mathcal{f}$ Dis Child 1991;145:1147-9.

10 Cooke R, Hollis B, Conner C, Watson D, Werkman S, Chesney R. Vitamin D and mineral metabolism in the very
low birth weight infant receiving 400 IU of vitamin D. $f$ Pediatr 1990;116:423-8.
11 Koo WW, Krug-Wispe S, Neylan M, Succop P, Oestreich AE, Tsang RC. Effect of three levels of vitamin D intake in $\mathrm{AE}$, Tsang RC. Effect of three levels of vitamin D intake in
preterm infants receiving high-mineral containing milk. $\mathcal{F}$ preterm infants receiving high-mineral cont

12 Demirian A, Goldstein H, Tanner JM. A new system of dental assessment. Hum Biol 1973;45:211-27.

13 Demirjian A, Goldstein H. New systems for dental maturity based on seven and four teeth. Ann Hum Biol 1976;3:41121.

14 Jensen $H$, Christiansen C, Lindbjerg IF, Munck O. The mineral content in bone measured by means of $27.5 \mathrm{keV}$ radiation from ${ }^{125}$ I. Acta Radiol Suppl 1972;313:214-20.

15 Karjalainen P. A method for determination of the mineral content and the mineral density in the distal radius using gamma ray attenuation. Annals of Clinical Research 1973;5:231-7.

16 Sievänen H, Oja P, Vuori I. Scanner-induced variability and quality assurance in longitudinal dual-energy X-ray quality assurance in longitudinal dual-energy X-ray
absorptiometry measurements. Med Phys 1994;21:1795805 .

17 Sievänen H, Kannus P, Nieminen V, Heinonen A, Oja P, Vuori I. Estimation of various mechanical characteristics of human bones using dual energy X-ray absorptiometry: methodology and precision. Bone 1996;18:S17-28.

18 Demirjian A, Levesque GJ. Sexual difference in dental development and prediction of emergence. $f$ Dent Res $1980 ; 59 ; 1110-22$

19 Garn SM, Lewis AB, Koski K, Polacheck DL. The sex difference in tooth calcification. F Dent Res 1958;37:561-7.

20 Haavikko K. The formation and the alveolar and clinical eruption of the permanent teeth. An orthopantomographic study. Proceedings of the Finnish Dental Society 1970;66:103-70.

21 Taranger J, Lichtenstein H, Svennberg-Redegren I. The somatic development of children in a Swedish urban community. A prospective longitudinal study. III. Dental development from birth to 16 years. Acta Paediatr Scand 1976;258(suppl):83-97.

22 Taranger J, Bruning B, Claesson I, Karlberg P, Landström T. Lindström B. The somatic development of children in a Swedish urban community. A prospective longitudinal study. V. A new method for the assessment of skeletal maturity: the MAT method (mean appearance time of bone stages). Acta Paediatr Scand 1976;258(suppl):10920.

23 Hatton ME. A measure of the effects of heredity and environment on eruption of the deciduous teeth. $\mathcal{F}$ Dent Res 1955;34:397-401.

24 Robinow M, Richard TW, Anderson M. The eruption of deciduous teeth. Growth 1942;6:127-33.

25 Nyström M, Haataja J, Kataja M, Evalahti M, Peck L, Kleemola-Kujala E. Dental maturity in Finnish children, estimated from the development of seven permanent mandibular teeth. Acta Odontol Scand 1986;44:193-8.

26 Kataja M, Nyström M, Aine L. Dental maturity standards in southern Finland. Proceedings of the Finnish Dental Society 1989;85:187-97.

27 Hamano Y, Hägg U. Inter-relationships among ages of emergence of teeth. A prospective longitudinal study of Swedish children from birth to 18 years. Eur $\mathcal{F}$ Orthod 1988;10:273-80.

28 Backström MC, Mäki R, Kuusela A-L, Sievänen H, Koivisto A-M, Ikonen RS, Mäki M. Randomized controlled trial of vitamin D supplementation on bone density and biochemical indices in preterm infants. Arch Dis Child 1999;80:F161-6

29 Backström MC, Mäki R, Kuusela A-L Sievänen H, Koivisto A-M, Koskinen $M$, Ikonen $R S$ and Mäki $M$. The long-term effect of early mineral, vitamin $\mathrm{D}$ and breast milk intake on bone mineral status in 9-11 years old children born prematurely. $f$ Pediatr Gastroenterol Nutr 1999;29:575-82. 